\title{
Interdiffusion-Induced Polarization-Independent Optical Gain of an InGaAs-InP Quantum-Well with Carrier Effects
}

\author{
Wallace C. H. Choy, Student Member, IEEE, E. Herbert Li, Senior Member, IEEE, \\ Michael C. Y. Chan, and Bernard L. Weiss, Senior Member, IEEE
}

\begin{abstract}
A theoretical study of the polarization-independent optical gain using group $V$ sublattice interdiffusion in InGaAs-InP quantum wells (QW's) is presented here. The reverse bias and carrier effects on the subband structures, transition energy, and optical gain of the interdiffused $Q W$ are discussed. The interdiffused $Q W$ structures are optimized in terms of their subband structure, carrier density, structural parameters, and properties of optical gain spectra. The results show that an optimized interdiffused $Q W$ structure can produce polarization-independent optical gain over a range of operation wavelengths around $1.5 \mu \mathrm{m}$, although the differential gain and linewidth enhancement factor are slightly degraded. The required tensile strain for the polarization-independent optical properties of a lattice-matched QW structure may be generated using interdiffusion. These results suggest that polarization-independent optical devices can be fabricated using interdiffusion in a lattice-matched InGaAsP QW structure.
\end{abstract}

Index Terms - Carrier effects, diffused quantum well, optical gain, optical polarization independence quantum well, reverse bias effects, quantum-well amplifier.

\section{INTRODUCTION}

D IFFERENT types of quantum wells (QW's) have been used as the active region of optical devices because of their enhanced optical properties, as compared to conventional bulk materials. However, quantum confinement effects break the symmetry of the electron-hole dipole and produce the TE and TM polarization dependence of the QW optical properties, including their optical gain, absorption coefficient, and refractive index when the incident light is parallel to the plane of the QW structure [1]. This is a well-known inherent feature of conventional QW's and a disadvantage for their use in optical communication systems. For strained QW materials, such as an InGaAsP QW, polarization independence is achieved by merging the heavy hole $(\mathrm{HH})$ and light hole ( $\mathrm{LH})$ by generating a tensile strain in the well. There are two possible ways to induce this tensile strain to produce polarizationindependent optical devices, such as optical amplifiers [2], [3], optical modulators [4], [5], and waveguides [6]: one can

Manuscript received May 19, 1998; revised February 18, 1999. This work was supported in part by the Hong Kong Research Grant Council earmarked grant. The work of W. C. H. Choy was supported by the Croucher Foundation.

W. C. H. Choy and B. L. Weiss are with the School of Electronic Engineering, Information Technology and Mathematics, University of Surrey, Guildford, Surrey GU2 5XH, UK.

E. H. Li and M. C. Y. Chan are with the Department of Electrical and Electronic Engineering, University of Hong Kong, Hong Kong.

Publisher Item Identifier S 0018-9197(99)04105-6. either tailor the sublattice compositions in the well of an asgrown QW structure [2], [4] or use an interdiffused QW to produce the tensile strain in the well [3], [5]. Previously, the polarization-independent electroabsorption InGaAsP interdiffused QW modulator was proposed using a subband model without valence band mixing and carrier effects [5]. With an extended band mixing model solved self-consistently with Poisson's equation, the details of the interdiffusion induced polarization independence in InGaAsP interdiffused QW's, including the effects of interdiffusion and free carriers on the subband structures, optical matrix elements, and optical gain of the interdiffused QW, are reported here. However, their effects on the polarization-independent interdiffused QW structures have not been analyzed previously.

In this paper, a theoretical study of the polarizationindependent gain of an InGaAs-InP QW using interdiffusion is carried out. The effects of interdiffusion on the subband structure, photon energy, and optical gain are analyzed. Taking into account free-carrier screening effects, the modification of the interdiffused QW optical properties due to different carrier densities, applied fields, and QW structure parameters (i.e., well width $L_{z}$ and atomic composition) are discussed. The polarization-independent gain of the optimized interdiffused QW structures is also presented.

\section{MODEL}

The model of the interdiffusion is group $\mathrm{V}$ two-phase interdiffusion [7], [8] where only the group $\mathrm{V}$ elements in InGaAsP are involved and the diffusion coefficients of these elements in the barriers and wells are different so that "two-phase" interdiffusion is obtained. For a QW heterostructure, where the InGaAs well is sandwiched between two InP barriers, the interdiffusion process can be represented by a set of diffusion equations for each species. This is defined as follows:

$$
\frac{\partial \zeta_{i}(z, t)}{\partial t}=D_{i} \frac{\partial^{2} \zeta_{i}(z, t)}{\partial z^{2}}
$$

where $z$ is the growth direction, $t$ is the annealing time, the subscript $i$ denotes the barrier for $|z| \geq L$ or the well for $|z|<$ $L, L$ is the position of the as-grown $\mathrm{QW}$ interface from the well center $(z=0)$, and $\zeta$ and $D$ are the concentration of the diffusion species and their diffusion coefficients, respectively. In solving this partial differential equation, two continuity conditions must be satisfied at the well-barrier interfaces (i.e., 
$z= \pm L)$, namely

$$
\left.\zeta_{\text {barrier }}(t)\right|_{z= \pm L}=\left.k \zeta_{\text {well }}(t)\right|_{z= \pm L}
$$

and

$$
\left.D_{\text {barrier }} \frac{\partial \zeta_{\text {barrier }}(t)}{\partial z}\right|_{z= \pm L}=\left.D_{\text {well }} \frac{\partial \zeta_{\text {well }}(t)}{\partial z}\right|_{z= \pm L} .
$$

Equation (2) models the discontinuous $(k \neq 1)$ concentration at the interface while (3) expresses the flux continuity. Further details have been thoroughly discussed elsewhere [8]. Equation (1) is solved using a finite difference method to obtain the concentration distribution of the diffused species after an annealing time $t$. This concentration ratio gives the spatial molar fraction (composition) of the interdiffused As concentration $\tilde{y}$ in the $\operatorname{In}_{x_{o}} \mathrm{Ga}_{1-x_{o}} \mathrm{As}_{\tilde{y}} \mathrm{P}_{1-\tilde{y}}$ interdiffused $\mathrm{QW}$ structure. The parameter $x_{O}$ is the concentration of the In atoms (Group III composition) and, since the interdiffusion only involves Group $\mathrm{V}$ composition, $x_{O}$ is a constant. The molar fractions $x_{O}$ and $\tilde{y}$ are used to determine the material parameters, including the carrier effective masses and potential profiles in modeling the QW subband structures. The induced tensile strain strongly depends on the extent of the interdiffusion. The various stages of interdiffusion can be obtained by varying $t$ and the temperature, where the temperature affects the diffusion coefficients.

The coupling of an $\mathrm{HH}$ and an $\mathrm{LH}$ is considered using the $4 \times 4$ Luttinger-Kohn Hamiltonian [9]. With effective mass theory, the Hamiltonian for the interdiffused QW valence band can be written as (4), shown at the bottom of the page, where

$$
\begin{aligned}
P= & \frac{\hbar^{2}}{2 m_{o}} \gamma_{1}\left(k_{x}^{2}+k_{y}^{2}-\frac{\partial^{2}}{\partial z^{2}}\right) \\
Q= & \frac{\hbar^{2}}{2 m_{o}} \gamma_{2}\left(k_{x}^{2}+k_{y}^{2}+2 \frac{\partial^{2}}{\partial z^{2}}\right) \\
V_{\mathrm{HH}}(z)= & \tilde{V}^{V}(z)-|e| F z+|e| \varphi(z) \\
& -\left(1-Q_{c}\right) S_{\perp}(x(z), \tilde{y}(z))+S_{/ / \mathrm{HH}}(x(z), \tilde{y}(z)) \\
V_{\mathrm{LH}}(z)= & \tilde{V}^{V}(z)-|e| F z+|e| \varphi(z) \\
& -\left(1-Q_{c}\right) S_{\perp}(x(z), \tilde{y}(z))+S_{/ / \mathrm{LH}}(x(z), \tilde{y}(z)) \\
\Omega= & -\frac{\hbar^{2}}{m_{o}}\left(\frac{\sqrt{3}}{2} \bar{\gamma}\left(k_{x}-i k_{y}\right)^{2}\right. \\
& \left.-\frac{\sqrt{3}}{4}\left(\gamma_{3}-\gamma_{2}\right)\left(k_{x}+i k_{y}\right)^{2}\right)
\end{aligned}
$$

and

$$
B=\sqrt{3} \frac{\hbar^{2}}{m_{o}} \gamma_{3}\left(k_{x}-i k_{y}\right)\left(-i \frac{\partial}{\partial z}\right)
$$

where $z$ denotes the growth direction, $\tilde{V}^{V}(z)$ is the diffused potential profile of the valence band, and $F$ and $\varphi(z)$ are the applied electric field and the potential due to carriers, respectively. $Q_{c}$ is the conduction band offset ratio. $S_{\perp}, S_{/ / \mathrm{HH}}$, and $S_{/ / \mathrm{LH}}$ are the biaxial component of strain and the splitting energy of the $\mathrm{HH}$ and $\mathrm{LH}$ induced by the uniaxial component of the strain, respectively, and the well center is defined as $z=$ 0 . Details of the relationships between $S_{\perp}, S_{/ / \mathrm{HH}}$, and $S_{/ / \mathrm{LH}}$ are given in [10]. Also, $m_{o}$ is the electron mass, $\gamma_{1}, \gamma_{2}$, and $\gamma_{3}$ are the Luttinger-Kohn parameters [11], where $\bar{\gamma}=\frac{1}{2}\left(\gamma_{2}+\gamma_{3}\right)$ and $k_{/ l}^{2}=k_{x}^{2}+k_{y}^{2}$. The fourfold degenerate block envelope functions that satisfy (4) have quantum numbers $-3 / 2,-1 / 2$, $1 / 2$, and $3 / 2(\mathrm{HH}= \pm 3 / 2, \mathrm{LH}= \pm 1 / 2)$. For simplicity, the axial approximation, which assumes that $\gamma_{2}=\gamma_{3}$, is applied to the $\Omega$ term [12], [13], and the $4 \times 4$ Hamiltonian is transformed into two $2 \times 2$ Hamiltonians, $H^{U}$ and $H^{L}$, using the framework in $[13]-[15]$

$$
H^{\delta}=\left[\begin{array}{cc}
P \pm Q+V_{\mathrm{HH}}(z) & \xi \\
\xi^{*} & P \mp Q+V_{\mathrm{LH}}(z)
\end{array}\right]
$$

where the superscript $\delta=U$ or $L$ and $\xi=\Omega+i|B|$. Equation (5) is solved using a finite difference method.

The conduction band at the $\Gamma$-valley $\left(k_{/ /}=0\right)$ is modeled using the one-dimensional, one particle Schrödinger-like equation [16] obtained from an envelope function approximation [17] through a BenDenial and Duke model [18]

$$
\begin{aligned}
\frac{-\hbar^{2}}{2} \frac{d}{d z}\left[\frac{1}{m^{*}(z)}\right. & \frac{d}{d z}+\tilde{V}^{C}(z)+|e| F z-|e| \varphi(z) \\
& \left.-Q_{c^{S}} S_{\perp}(x(z), \tilde{y}(z))\right] \psi_{a}^{C}(z)=E_{a} \psi_{a}^{C}(z)
\end{aligned}
$$

where $\tilde{V}^{C}(z)$ is the diffused potential profile of the conduction band, $m^{*}(z)$ is the effective mass of the electron, and $\psi_{a}^{C}(z)$ represents the envelope wavefunction of an electron to the $a$ th confined subband state with an energy $E_{a}$.

The potential due to carriers $\varphi(z)$ can be obtained by solving Poisson's equation self-consistently with (5) and (6)

$$
\frac{d^{2}}{d z^{2}} \varphi(z)=-\frac{|e|}{\varepsilon}\left[p(z)-n(z)+N_{D}(z)-N_{A}(z)\right]
$$

where $\varepsilon$ is the dielectric constant of InGaAsP and $N_{D}$ and $N_{A}$ are the densities of ionized donors and acceptors, respectively. For simplification, $N_{D}$ and $N_{A}$ are assumed to be zero. The carrier densities $p(z)$ and $n(z)$ are given as

$$
\begin{aligned}
p(z)=\frac{1}{2 \pi} \int_{k_{/ /}} \sum_{b} \sum_{i}\left|\psi_{i, b}^{V}(z)\right|^{2} & \\
& \times\left(1-f^{V}\left(E_{b}^{V}\left(k_{/ /}\right)\right)\right) k_{/ /} d k_{/ /}
\end{aligned}
$$

$$
\left(\begin{array}{cccc}
P+Q+V_{\mathrm{HH}}(z) & \Omega & -B & 0 \\
\Omega^{*} & P-Q+V_{\mathrm{LH}}(z) & 0 & B \\
-B^{*} & 0 & P-Q+V_{\mathrm{LH}}(z) & \Omega \\
0 & B^{*} & \Omega^{*} & P+Q+V_{\mathrm{HH}}(z)
\end{array}\right)
$$


and

$$
\begin{aligned}
n(z)= & \frac{m_{e} k_{B} T}{\pi \hbar^{2}} \sum_{a}\left|\psi_{a}^{C}(z)\right|^{2} \\
& \times \ln \left[1+\exp \left(\frac{F_{n}-\left(E_{a}^{C}-E_{1}^{C}\right)}{k_{B} T}\right)\right]
\end{aligned}
$$

where $k_{B}$ and $T$ are the Boltzmann constant and temperature, respectively, $F_{n}$ is the quasi-Fermi level for the electron in the conduction subband, $f^{V}\left(E_{b}^{V}\right)$ is the electron Fermi distribution function of the $b$ th valence subband, and $\psi_{i, b}^{V}$ is the $b$ th envelope function in the valence subband where the subscript $i$ is the quantum number of the fourfold degenerate hole states.

The optical gain is modeled using the density of state approach

$$
\begin{aligned}
g(E)= & \sum_{a b} \frac{2 \pi q^{2} \hbar}{(2 \pi)^{2} n_{R} \varepsilon_{o} c m_{o}^{2}(2 L) E} \int d^{2} k_{/ /}\left|\widehat{e} \bullet \vec{P}_{a b}\left(k_{/ /}\right)\right|^{2} \\
& \bullet \delta\left(E_{a}^{C}\left(k_{/ /}\right)-E_{b}^{V}\left(k_{/ /}\right)-E\right) \\
& \times\left[f^{C}\left(E_{a}^{C}\left(k_{/ /}\right)\right)-f^{V}\left(E_{b}^{V}\left(k_{/ /}\right)\right)\right]
\end{aligned}
$$

where $c, n_{R}$, and $q$ are the speed of light in vacuum, refractive index, and electric charge, respectively, $E$ is the photon energy, $\vec{P}_{a b}$ is the optical matrix element for a transition between the $a$ th electron subband and the $b$ th valence subband, $\hat{e}$ is a unit vector along the polarization direction of the optical electric field, and $f^{C}\left(E_{a}^{C}\right)$ is the electron Fermi distribution function in the $a$ th conduction subband. The squared optical matrix element $\left|\hat{e} \bullet \vec{P}_{a b}\right|^{2}$ for TE mode polarization is

$$
\begin{aligned}
\mid \hat{e} \bullet & \left.\vec{P}_{a b}\left(k_{/ /}\right)\right|^{2} \\
= & |\langle x|P| s\rangle|^{2} \bullet\left[\frac{1}{2}\left(\left|\left\langle\psi_{a}^{C} \mid \psi_{3 / 2, b}^{V}\right\rangle\right|^{2}+\left|\left\langle\psi_{a}^{C} \mid \psi_{-3 / 2, b}^{V}\right\rangle\right|^{2}\right)\right. \\
& \left.+\frac{1}{6}\left(\left|\left\langle\psi_{a}^{C} \mid \psi_{-1 / 2, b}^{V}\right\rangle\right|^{2}+\left|\left\langle\psi_{a}^{C} \mid \psi_{1 / 2, b}^{V}\right\rangle\right|^{2}\right)\right] \cdot
\end{aligned}
$$

For TM mode polarization,

$$
\begin{aligned}
\left|\hat{e} \bullet \vec{P}_{a b}\left(k_{/ /}\right)\right|^{2}= & \frac{3}{2}|\langle x|P| s\rangle|^{2} \bullet\left[\left(\left|\left\langle\psi_{a}^{C} \mid \psi_{-1 / 2, b}^{V}\right\rangle\right|^{2}\right.\right. \\
& \left.+\left|\left\langle\psi_{a}^{C} \mid \psi_{1 / 2, b}^{V}\right\rangle\right|^{2}\right) .
\end{aligned}
$$

In determining the momentum matrix elements $\langle x|P| s\rangle$, the expression

$$
\left|\left\langle x\left|P_{x}\right| s\right\rangle\right|^{2}=\frac{m_{o}^{2}}{2 m_{e}} \frac{E_{g}\left(E_{g}+\Delta\right)}{E_{g}+\frac{2}{3} \Delta}
$$

is used where $\Delta$ is the spin-orbit splitting energy, $E_{g}$ is the bandgap energy, and $m_{e}$ is the electron effective mass.

The gain with a spectral broadening $\left(G\left(E^{\prime}\right)\right)$ of a single interdiffused QW structure at a transition energy $E^{\prime}$ is written as

$$
G\left(E^{\prime}\right)=\int d E^{\prime} g\left(E^{\prime}\right) L\left(E-E^{\prime}\right)
$$

where $L\left(E-E^{\prime}\right)$ is the Lorentzian line-broadening function. The differential gain $d g / d N$ is calculated using the following

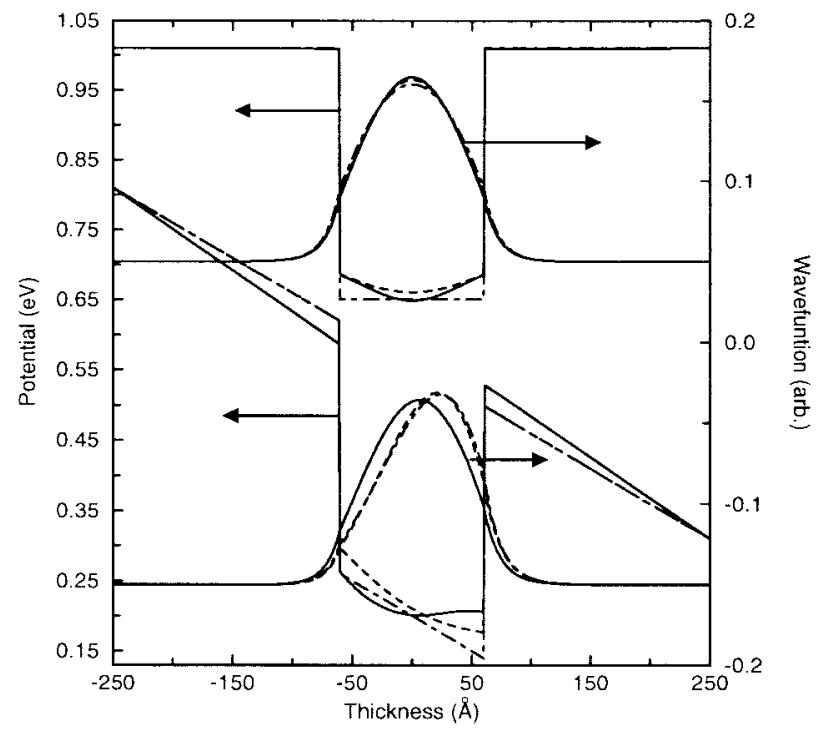

Fig. 1. The conduction band profile and wavefunction of fundamental eigenstate of the $\mathrm{In}_{0.53} \mathrm{Ga}_{0.47} \mathrm{As}-\mathrm{InP}$ interdiffused QW with $L_{z}=120 \AA$, a carrier density of $5 \times 10^{12} \mathrm{~cm}^{-2}$, and $F=0$ and $100 \mathrm{kV} / \mathrm{cm}$ at $k_{/ /}=0$; as-grown QW (dot-dash line), interdiffused QW with $t=0.5 \mathrm{~h}$ and without the carrier effects (dashed line), and interdiffused QW with $t=0.5 \mathrm{~h}$ and the carrier effects (solid line).

expression:

$$
\frac{d g}{d N}(E, n)=\lim _{\Delta N \rightarrow 0} \frac{G(E, N+\Delta N)-G(E, N)}{\Delta N}
$$

where $N$ is the carrier density. The linewidth enhancement factor $\alpha$, defined as the ratio of the change of refractive index with respect to the carrier density to the change of the optical gain with respect to the carrier density, is given as

$$
\alpha=-\frac{4 \pi}{\lambda} \frac{d n_{R} / d N}{d g / d N}
$$

where $\lambda$ is the photon wavelength and $d n_{R} / d N$ is obtained from $d g / d N$ using the Kramers-Krönig transformation.

\section{RESULTS AND DISCUSSION}

All InGaAs-InP interdiffused QW structures studied here are considered to be annealed at $750{ }^{\circ} \mathrm{C}$. The diffusion coefficient of the well and barrier materials, as well as their concentration ratios, are taken from a typical structure [8]. In modeling the optical gain of an interdiffused QW structure, the full-width at half-maximum (FWHM) of the lineshape function is assumed to be $10 \mathrm{meV}$. Due to band mixing effects, the $\mathrm{HH}$ and LH states discussed here are the states with dominant heavy-hole and light-hole characteristics, respectively.

\section{A. Effects of Interdiffusion}

In group $\mathrm{V}$ two-phase interdiffusion of $\operatorname{In}_{0.53} \mathrm{Ga}_{0.47} \mathrm{As}-\mathrm{InP}$ interdiffused QW structures with $L_{z}=120 \AA$ and a carrier density of $5 \times 10^{12} \mathrm{~cm}^{-2}, P$ diffuses from the well to the barrier while As diffuses in the reverse direction. As a result, the potential profile moves up and the well bottom becomes rounded (see Fig. 1). Although the wavefunction does not change significantly, the transition energy generally increases 
TABLE I

The EfFects of InTERdiffusion and CaRRIERs ON THE C1-HH1 TRANSITION Energy and TE Mode Gain Peak of the $\mathrm{In}_{0.53} \mathrm{Ga}_{0.47}$ As-InP QW with A CARRIER Density of $5 \times 10^{-12} \mathrm{~cm}^{-2}$ AT $k_{/ /}=0$. The CASES With AND Without the Carrier Effects Are Denoted by wC and woc, Respectively, GP Denotes the Gain Peak, and $t$ Is the Annealing Time

\begin{tabular}{|c|c|c|c|c|c|}
\hline case & $\mathrm{Cl}$ & $\mathrm{HH} 1$ & $\mathrm{Cl}-\mathrm{HHI}$ & GP & $\lambda$ \\
\hline $\mathrm{F}=0 \mathrm{kV} / \mathrm{cm}$ & $(\mathrm{eV})$ & $(\mathrm{eV})$ & $(\mathrm{eV})$ & $\left(\mathrm{cm}^{-1}\right)$ & $(\mathrm{eV})$ \\
\hline$t=0, w o c$ & 0.4842 & 0.3054 & 0.7896 & 1322 & 0.8176 \\
\hline $\mathrm{t}=0, \mathrm{wc}$ & 0.4785 & 0.3116 & 0.7901 & 1305 & 0.8170 \\
\hline $\mathrm{t}=0.5$, woc & 0.4980 & 0.3192 & 0.8171 & 1270 & 0.8401 \\
\hline $\mathrm{t}=0.5, \mathrm{wc}$ & 0.4900 & 0.3283 & 0.8183 & 1243 & 0.8413 \\
\hline$t=1$, woc & 0.5062 & 0.3284 & 0.8346 & 1213 & 0.8565 \\
\hline$t=1, w c$ & 0.5001 & 0.3352 & 0.8353 & 1189 & 0.8573 \\
\hline \multicolumn{6}{|l|}{$\mathrm{F}=100 \mathrm{kV} / \mathrm{cm}$} \\
\hline $\mathrm{t}=0$, woc & 0.4760 & 0.2808 & 0.7568 & 617 & 0.8058 \\
\hline $\mathrm{t}=0, \mathrm{wc}$ & 0.4794 & 0.3067 & 0.7861 & 1075 & 0.8171 \\
\hline $\mathrm{t}=0.5$, woc & 0.4904 & 0.3003 & 0.7907 & 629 & 0.8297 \\
\hline $\mathrm{t}=0.5, \mathrm{wc}$ & 0.4899 & 0.3253 & 0.8152 & 1065 & 0.8402 \\
\hline $\mathrm{t}=1$, woc & 0.4981 & 0.3069 & 0.8050 & 607 & 0.8450 \\
\hline$t=1, w c$ & 0.5006 & 0.3313 & 0.8319 & 1082 & 0.8559 \\
\hline
\end{tabular}

when diffusion time $(t)$ increases from 0 to $1 \mathrm{~h}$ for both $F=0$ and $100 \mathrm{kV} / \mathrm{cm}$, as shown in Table I.

The transitions between the first electron and the first $\mathrm{HH}$ $(\mathrm{C} 1-\mathrm{HH} 1)$ and between the first electron and the first $\mathrm{LH}$ (C1-LH1) at $k_{/ /}=0$ are shown in Fig. 2(a) where free-carrier screening effects have been included (see the details below). For the as-grown QW $(t=0)$, the HH1 and LH1 states are clearly separated. When $t$ increases, the splitting energy reduces due to the compensation of the interdiffusion-induced shear tensile strain generated in $\mathrm{HH} 1$ and LH1. At $t=1.4 \mathrm{~h}$, an average tensile strain of $0.275 \%$ is generated which makes the transitions merge. For larger $t$, the tensile strain is so large that the $\mathrm{HH} 1$ and $\mathrm{LH} 1$ transitions cross over, i.e., the transition energy of $\mathrm{C} 1-\mathrm{HH} 1$ becomes greater than that of $\mathrm{C} 1-\mathrm{LH} 1$. Consequently, the polarization-independent transition with a value of $0.841 \mathrm{eV}$ can be obtained at $t=1.4 \mathrm{~h}$.

The interdiffusion also changes the dispersion of the LH1 and HH1 subbands in $k_{/ /}$space, as shown in Fig. 2(b), when the energy of the spin-up and spin-down carriers keep close together at $k_{/ /} \neq 0$ for both HH1 and LH1. During interdiffusion, although the induced hydrostatic tensile strain reduces the transition energy, the modification of the interdiffused QW compositions dominate the change of the transition energy so that the resultant $\mathrm{C} 1-\mathrm{HH} 1$ and $\mathrm{C} 1-\mathrm{LH} 1$ transition energies increase. Therefore, the complete HH1 and LH1 subbands at $k_{/ /} \neq 0$ move downwards, as shown in Fig. 2(b). This suggests that interdiffusion can be used to adjust the operating wavelength of an optical device.

\section{B. Carrier Effects with Reverse Bias}

The free-carrier screening effects on the $\operatorname{In}_{0.53} \mathrm{Ga}_{0.47} \mathrm{As}-\mathrm{InP}$ interdiffused QW structure with $L_{z}=120 \AA, t=0.5 \mathrm{~h}$, and a carrier density of $5 \times 10^{12} \mathrm{~cm}^{-2}$ are best demonstrated by the potential profile and the fundamental wavefunction of the interdiffused QW with and without free-carrier screening effects (see Fig. 1). With carrier effects and $F=0$, the round-shaped well bottom of the interdiffused QW is pressed down, although there is no significant change in barriers and

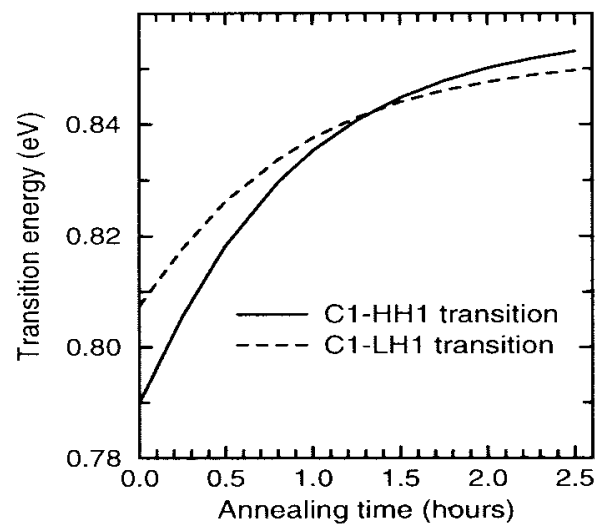

(a)

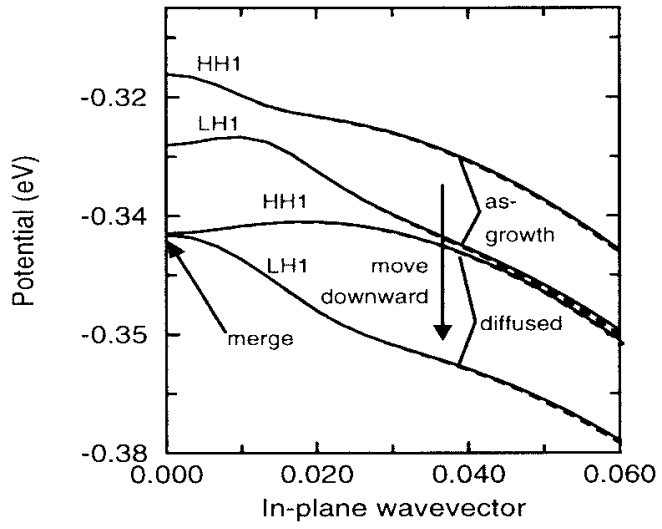

(b)

Fig. 2. (a) The transition energy at $k_{/ /}=0$ and (b) the valence subband dispersion in $k_{/ /}$space of the $\operatorname{In}_{0.53} \mathrm{Ga}_{0.47} \mathrm{As}$ - InP interdiffused QW with $L_{z}=120 \AA$ and a carrier density of $5 \times 10^{12} \mathrm{~cm}^{-2}$.

well-barrier interfaces. This results in a slight improvement of the wavefunction confinement and the peak of the wavefunction increases slightly, as compared to the interdiffused QW without the carrier effects. For an applied field $(F)$ of $100 \mathrm{kV} / \mathrm{cm}$, the carrier effects are more intuitive, the potential profile of the well bottom becomes bumpy, and the effects of reverse bias are reduced. Therefore, the wavefunction shifts toward the center of the interdiffused QW, i.e., the confinement of the wavefunction improves considerably as compared to the interdiffused QW without free-carrier screening effects.

The $\mathrm{C} 1-\mathrm{HH} 1$ transition energy increases when the carrier effects are considered in all interdiffused QW's with $t=0$ to $1 \mathrm{~h}$ and $F=0$, which is due primarily to the increase of the HH1 energy as the $\mathrm{C} 1$ energy generally decreases (see Table I). This suggests that the difference in the transition energy with and without carrier effects is dominated by the change of HH1. Similar results for the C1-HH1 transition energy are also obtained for $F=100 \mathrm{kV} / \mathrm{cm}$. However, here the increase in the transition energy with and without carrier effects is $>20 \mathrm{meV}$ for all interdiffused QW's with $t=0$ to $1 \mathrm{~h}$ as compared to $1-2 \mathrm{meV}$ in the interdiffused QW's at $F=0$. Nevertheless, the variation of the transition energy due to carrier effects is still dominated by the HH1 subband. As shown in Fig. 3, at $F=100 \mathrm{kV} / \mathrm{cm}$, the interdiffused QW valence subbands for $t=0.5 \mathrm{~h}$ increase when the carrier 


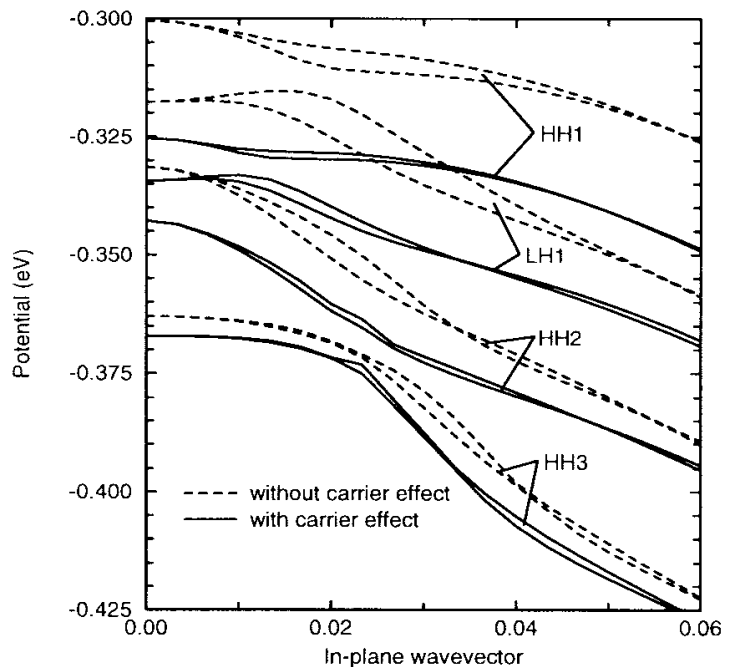

Fig. 3. The valence subband dispersion in $k_{/ /}$space of the $\mathrm{In}_{0.53} \mathrm{Ga}_{0.47}$ As-InP interdiffused QW with $L_{z}=120 \AA$, a carrier density of $5 \times 10^{12} \mathrm{~cm}^{-2}$, and $F=100 \mathrm{kV} / \mathrm{cm}$; with the carrier effects (solid line) and without the carrier effects (dash line).

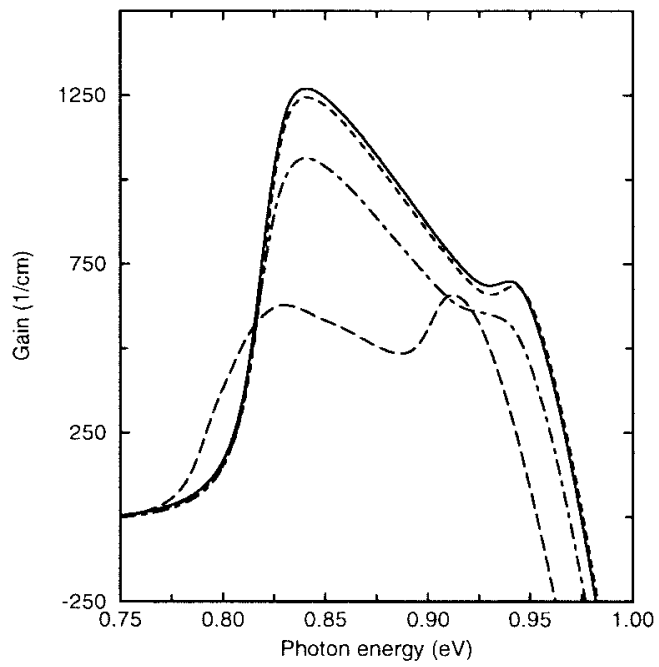

Fig. 4. The TE gain spectrum of the $\mathrm{In}_{0.53} \mathrm{Ga}_{0.47} \mathrm{As}-\mathrm{InP}$ interdiffused QW structure with $t=0.5 \mathrm{~h}$ and applied fields; $F=0$ and without the carrier effects (solid lines), $F=0$ and with carrier effects (dashed lines), $F=100$ $\mathrm{kV} / \mathrm{cm}$ and without the carrier effects (long dashed lines), and $F=100$ $\mathrm{kV} / \mathrm{cm}$ and with the carrier effects (dot-dash lines).

effects are considered, i.e., all transition energies increase. Since the screening effects of free carriers reduce the effect of reverse bias, spin splitting generally reduces. Consequently, the modification of the transition energy with and without carrier effects is insensitive to the extent of the interdiffusion, as shown in Table I, and carrier effects cause a blue shift of the transition energy which is dominated by the variation of the $\mathrm{HH}$ subbands.

The TE optical gain spectra of the interdiffused QW with $t=0.5 \mathrm{~h}$ for various applied fields are shown in Fig. 4. At $F=0$, the difference of the optical gain spectra with and without carrier effects is small. However, at $F=100 \mathrm{kV} / \mathrm{cm}$, the optical gain of the interdiffused QW with the carrier effects increases significantly and blue shifts, as compared to the interdiffused QW without the carrier effects. For interdiffused

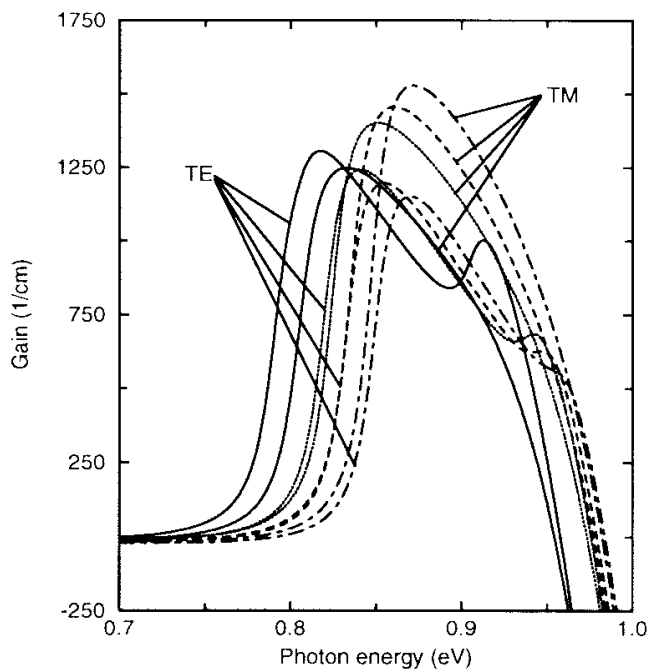

Fig. 5. TE and TM gain spectra of the $\mathrm{In}_{0.53} \mathrm{Ga}_{0.47}$ As-InP interdiffused QW with a carrier density of $5 \times 10^{12} \mathrm{~cm}^{-2}$ and different annealing times; $t=0$ (solid lines), $t=0.5 \mathrm{~h}$ (dotted lines), $t=0.8 \mathrm{~h}$ (dashed lines), and $t=2 \mathrm{~h}$ (dot-dash lines).

QW's with $t=0$ to $1 \mathrm{~h}$, with carrier effects, the gain peak at $F=0$ is reduced slightly as compared to the absence of carrier effects (see Table I). On the contrary, at $F=100$ $\mathrm{kV} / \mathrm{cm}$, the interdiffused QW gain peak increases substantially due to the weakening of reverse bias effects and improvement of the wavefunction confinement (see Fig. 1).

\section{Polarization-Independent Gain}

Group V two-phase interdiffusion can be used to modify the magnitude and spectral position of the interdiffused QW optical gain and its polarization-independent properties. It should be noted that the lower energy side of a gain spectrum is denoted as its front side to simplify the discussion.

The TE and TM gain spectra of the $\operatorname{In}_{0.53} \mathrm{Ga}_{0.47} \mathrm{As}-\mathrm{InP}$ interdiffused QW structure with $L_{z}=120 \AA$ blue shift when $t$ increases from $t=0$ to $2 \mathrm{~h}$ (see Fig. 5). At the same time, the lower energy side of the TE and TM gain spectra merge at a suitable $t$, due to the interdiffusion-induced tensile strain. Initially, the energy of the front side of the TE gain spectrum is lower than that of the TM one for the as-grown QW structure because $\mathrm{C} 1-\mathrm{HH} 1$ is lower than $\mathrm{C} 1-\mathrm{LH} 1$. When $t$ increases, the difference of the front-side energy reduces, and at $t=0.8 \mathrm{~h}$ the TE and TM gain spectra overlap, i.e., polarization-independent gain is achieved over a wavelength range from $0.78 \mathrm{eV}$ to 0.84 $\mathrm{eV}$. As $t$ increases further to $2 \mathrm{~h}$, the TM front-side energy is lower than that of the TE mode because the lowest transition changes from $\mathrm{C} 1-\mathrm{HH} 1$ to $\mathrm{C} 1-\mathrm{LH} 1$.

It is worth noting that the required $t$ to achieve polarizationindependent gain in the $x=0.53$ structure is $\sim 0.8 \mathrm{~h}$ (see Fig. 5), which is less than that predicted for the polarizationindependent transition $(1.4 \mathrm{~h})$ [see Fig. 2(a)]. This time difference arises since the variation of the transition energy only depends on the modification of the subbands due to interdiffusion. However, the optical gain depends on the lineshape broadening factor and the different $\mathrm{HH}$ and $\mathrm{LH}$ polarization factors in the optical matrix elements, see (11) and 
(a)

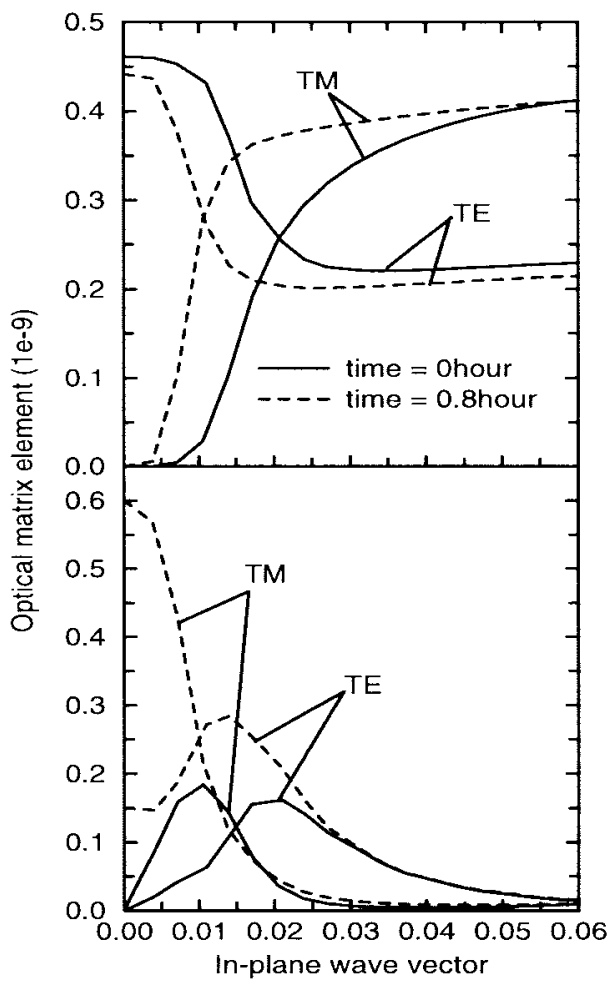

Fig. 6. Optical matrix element of the $\mathrm{In}_{0.53} \mathrm{Ga}_{0.47} \mathrm{As}-\mathrm{InP}$ interdiffused QW with $t=1 \mathrm{~h}$. (a) C1-HH1. (b) C1-LH1.

(12), which vary with the extent of interdiffusion. Therefore, the time required to produce polarization-independent gain is different than the time required for polarization-independent transitions, as shown in Fig. 2(a).

The magnitude of the TE and TM gain peaks also change when $t$ increases (see Fig. 5). When $t=0$, the TE gain peak is larger than the TM one for the as-grown QW structure. However, when $t$ increases, the TM gain peak grows and becomes the larger one, which can be explained by the variation of optical matrix elements, as shown in Fig. 6. For the as-grown $\mathrm{QW}$ with $t=0$, the TE optical matrix elements of $\mathrm{C} 1-\mathrm{HH} 1$ is at its maximum when $k_{/ /} \rightarrow 0$ [see Fig. 6(a)]. However, the TM optical matrix elements of both C1-HH1 and $\mathrm{C} 1-\mathrm{LH} 1 \rightarrow 0$ when $k_{/ /} \rightarrow 0$ so that the TE gain peak is greater than the TM gain peak at $t=0$. For $t=0.8 \mathrm{~h}$, the TE optical matrix elements of $\mathrm{C} 1-\mathrm{HH} 1$ reduce when the TM optical matrix elements of $\mathrm{C} 1-\mathrm{LH} 1$ increase to their maximum when $k_{/ /} \rightarrow 0$ (see Fig. 6). This is because the interdiffusioninduced shear tensile strain in the well increases, which moves the LH1 subband closer to the lowest subband energy [19]. Consequently, the TM optical gain peak increases to exceed the TE one after interdiffusion. These results suggest that the optical mode of the highest gain peak can be adjusted from TE to TM using interdiffusion. In addition, the threshold current of a QW laser operated at a gain peak can be increased for TE mode operation or decreased for TM mode operation using interdiffusion.

For the interdiffused QW with $t=0.8 \mathrm{~h}$, the TE optical matrix elements of $\mathrm{C} 1-\mathrm{HH} 1$ are smaller over the entire range of $k_{/ /}$from 0 to 0.06 as compared to that of the as-grown QW $(t=0)$. However, the TM optical matrix elements of

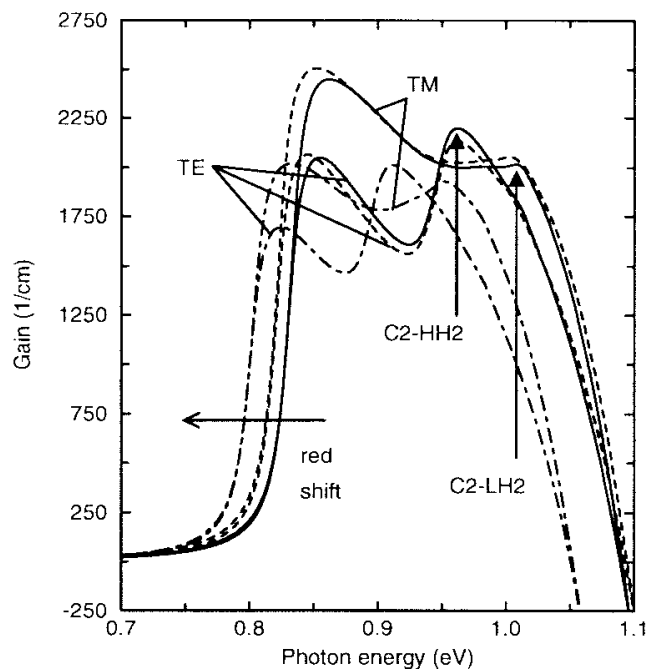

Fig. 7. TE and TM gain spectra of $\operatorname{In}_{x} \mathrm{Ga}_{1-x}$ As-InP interdiffused QW with a carrier density of $9 \times 10^{12} \mathrm{~cm}^{-2}$; interdiffused QW with $x=0.53$, $L_{z}=120 \AA$ and $t=0.8 \mathrm{~h}$ (solid lines), interdiffused QW with $x=0.51$, $L_{z}=120 \AA$ and $t=0.24 \mathrm{~h}$ (dash lines) and interdiffused $\mathrm{QW}$ with $x=0.51, L_{z}=140 \AA$ and $t=\sim 0.1 \mathrm{~h}$ (dot-dash lines).

the interdiffused QW C1-HH1 increases in the range of $k_{/ /}$ as compared to that of the as-grown QW (see Fig. 6). This is because the HH1 subband has more light-hole characteristics due to the band mixing effects and the LH1 subband energy moves closer to the lowest subband energy for $t=0.8 \mathrm{~h}$. The variations of the optical matrix elements contribute to weakening and strengthening the TE and TM optical gain, respectively, though the corresponding photon energy changes when $k_{/ /}$increases.

When the carrier density of the $\operatorname{In}_{0.53} \mathrm{Ga}_{0.47} \mathrm{As}-\mathrm{InP}$ interdiffused QW structure with $L_{z}=120 \AA$ and $t=0.8$ hour increases from $5 \times 10^{12} \mathrm{~cm}^{-2}$ to $9 \times 10^{12} \mathrm{~cm}^{-2}$, the complete TE and TM gain spectra increase, as shown in Fig. 7, because the quasi-Fermi level increases. In addition, the TE and TM gain peaks corresponding to the high-order transitions are enhanced when the carrier density increases (see Fig. 6). The second peak of TE (TM) optical gain at $\approx 0.96$ $\mathrm{eV}(\approx 1.01 \mathrm{eV})$ due to the $\mathrm{C} 2-\mathrm{HH} 2(\mathrm{C} 2-\mathrm{LH} 2)$ transitions increases when the carrier density increases to $9 \times 10^{12} \mathrm{~cm}^{-2}$. Consequently, the increase of the carrier density produces a higher polarization-independent gain. Using the carrier density of $9 \times 10^{12} \mathrm{~cm}^{-2}$ in the $\mathrm{In}_{0.53} \mathrm{Ga}_{0.47}$ As-InP interdiffused QW structure, polarization-independent gains as high as $\sim 1750$ $\mathrm{cm}^{-1}$ can be obtained for $t=0.8 \mathrm{~h}$.

Polarization-independent gain up to $2100 \mathrm{~cm}^{-1}$ can be obtained in the gain peak region at a specific photon energy of $0.83 \mathrm{eV}$ using an $x=0.53$ interdiffused QW structure after annealing for $0.2 \mathrm{~h}$ (see Fig. 8). This polarization-independent gain is only obtained at a single wavelength where the TE and TM mode gain spectra cross each other.

\section{Effects of $Q W$ Parameters}

The as-grown QW parameters that can be used to modify the gain spectra are the sublattice composition and $L_{z}$. When the In content decreases from $x=0.53$ to $x=0.51$, the 


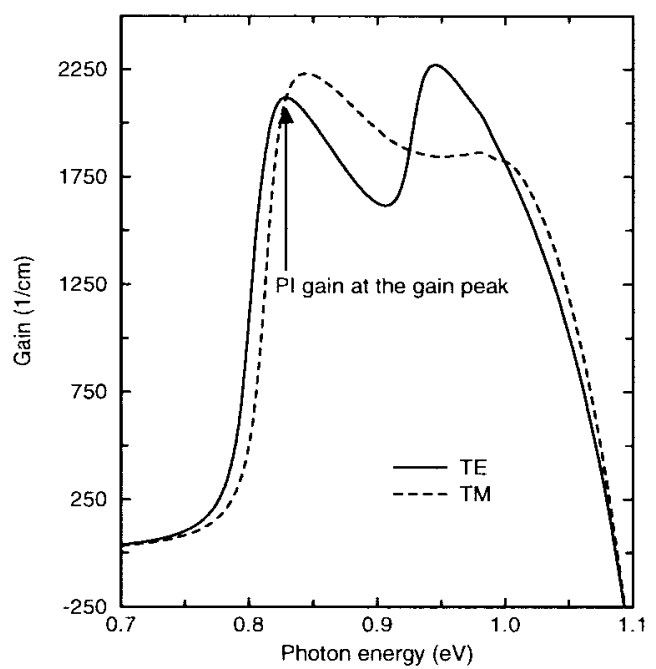

Fig. 8. TE (solid line) and TM (dashed line) gain spectra of the $\mathrm{In}_{0.53} \mathrm{Ga}_{0.47}$ As-InP interdiffused QW with $L_{z}=120 \AA$, $t=0.2 \mathrm{~h}$, a carrier density of $9 \times 10^{12} \mathrm{~cm}^{-2}$, showing that polarization-independent (PI) gain occurs near the gain peak.

required $t$ falls from 0.8 to $\sim 0.24 \mathrm{~h}$ because part of the tensile strain required to produce polarization-independent operation has already been built-in during sample growth, i.e., $0.15 \%$ strain in an $\mathrm{In}_{0.51} \mathrm{Ga}_{0.49} \mathrm{As}-\mathrm{InP} \mathrm{QW}$. Since a shorter time is needed for an $x=0.51$ interdiffused $\mathrm{QW}$, less $P$ diffuses into the well region so that the $\mathrm{C} 1-\mathrm{HH} 1$ and $\mathrm{C} 1-\mathrm{LH} 1$ transition energies decrease, which results in a red-shift of the front side of the optical gain spectra (see Fig. 7). By increasing $L_{z}$ from 120 to $140 \AA$, $t$ can be reduced further to $\sim 0.1 \mathrm{~h}$ since the splitting energy of $\mathrm{HH} 1$ and $\mathrm{LH} 1$ reduces when $L_{z}$ increases. Since $t$ and $L_{z}$ decrease and increase, respectively, the operating photon energy can be further reduced and the polarization-independent gain at $0.8 \mathrm{eV}$ increases to $\sim 1000$ $\mathrm{cm}^{-1}$. It is valuable to note that, in the case of interdiffused QW with $x=0.51$ and $L_{z}=120 \AA$, the required $t$ of 0.24 $\mathrm{h}$ for polarization-independent gain is shorter than that of 0.5 $\mathrm{h}$ for the polarization-independent transition. This agrees with the result of the $x=0.53$ interdiffused $\mathrm{QW}$, as discussed in Section C.

The differential gain indicated the rate of increase of optical gain with the injected carrier density is shown in Fig. 9. When $t$ increases, the differential gain decreases. For the $\mathrm{In}_{0.51} \mathrm{Ga}_{0.49} \mathrm{As} / \mathrm{InP}$ interdiffused QW with $L_{z}=120 \AA$, the differential gain for the TE and TM modes are very similar for a short time between 0.2 and $0.25 \mathrm{~h}$. The rate of increase of the polarization independent differential gain is about $0.13 \times 10^{-9}$ $\mathrm{cm}$ at $t=0.25 \mathrm{~h}$. When $t$ increases, the TM differential gain is larger than that for the TE one but both saturate for long times, greater than $1 \mathrm{~h}$. Consequently, although the differential gain decreases and the performance degrades when $t$ increases, only a short time $(0.24 \mathrm{~h})$ is required to produce polarization-independent gain for the $x=0.51$ interdiffused QW [see Fig. 9(a)] so that the degradation is not significant. Similar features can also be obtained for the $x=0.53$ interdiffused $\mathrm{QW}$ with $t=0.8 \mathrm{~h}$ so that the differential gain of this structure is $\sim 0.15 \times 10^{-9} \mathrm{~cm}$, although it is not detailed here. (a)

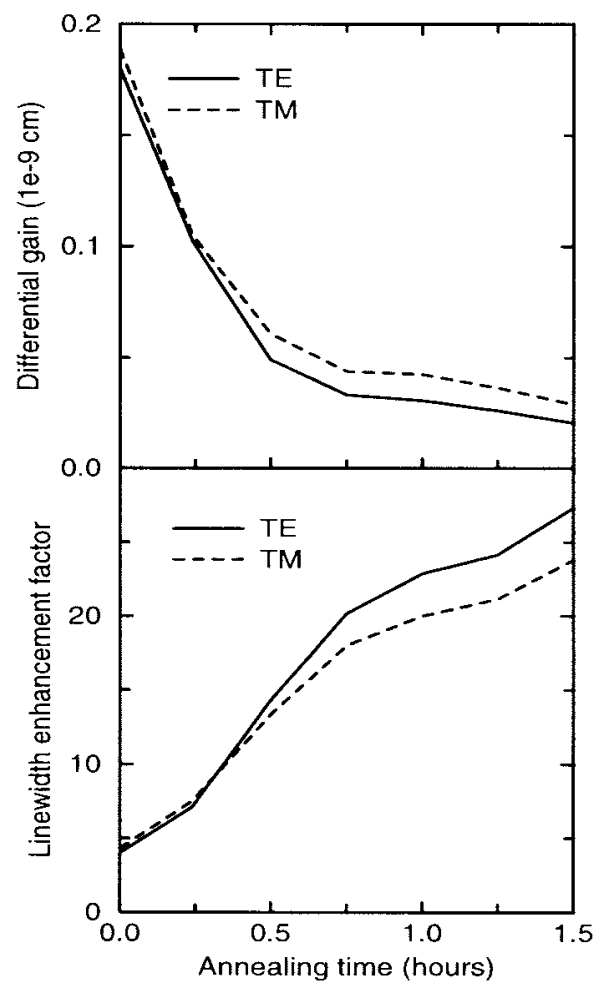

Fig. 9. (a) The differential gain and (b) the linewidth enhancement factor of the $\operatorname{In}_{0.51} \mathrm{Ga}_{0.49} \mathrm{As}-\mathrm{InP}$ interdiffused QW with $L_{z}=120 \AA$ and a carrier density of $9 \times 10^{12} \mathrm{~cm}^{-2}$ for a photon energy of $0.82 \mathrm{eV}$; TE (solid line) and TM (dashed line).

As shown in Fig. 9(b), the linewidth enhancement factor $\alpha$ increases when $t$ increases. However, since only a short $t$ is needed for the polarization-independent gain, $\alpha$ does not increase significantly. For the $x=0.51$ interdiffused $\mathrm{QW}$ with $L_{z}=120 \AA$ and $t=0.24 \mathrm{~h}, \alpha$ is 8 but is $\sim 5$ for the asgrown one at a photon energy of $0.82 \mathrm{eV}$. For the $x=0.51$ interdiffused $\mathrm{QW}$ with $L_{z}=140 \AA$ and $t=0.1 \mathrm{~h}, \alpha$ is 7.5 whereas it is $\sim 7$ for the as-grown one at a photon energy of $0.8 \mathrm{eV}$. Therefore, there is an acceptable degradation in both the differential gain and $\alpha$ for the optimized interdiffused QW structures.

\section{E. Effects of Reverse Bias}

The field-induced quantum-confined Stark shift of the optical gain of the $\operatorname{In}_{0.53} \mathrm{Ga}_{0.47}$ As-InP interdiffused QW's with $L_{z}=120 \AA$ and $t=0$ and $0.8 \mathrm{~h}$ is shown in Fig. 10. For $F=100 \mathrm{kV} / \mathrm{cm}$, both the as-grown QW and the interdiffused QW can provide gain switching. The operating wavelength can be adjusted using interdiffusion, although the change of the interdiffused QW gain with applied field at the TE gain peak $\left(250 \mathrm{~cm}^{-1}\right)$ is weaker than that of the as-grown $\mathrm{QW}$ $\left(500 \mathrm{~cm}^{-1}\right)$.

Even though the polarization-independent gain can be maintained under an applied field of $F=0$ and $100 \mathrm{kV} / \mathrm{cm}$, polarization-independent gain and polarization-independent gain switching may not be very important for lasers because lasers are usually linearly polarized and the presence of polarization-independent gain may make the laser less stable due to mode hopping [20]. However, polarization 
(a)

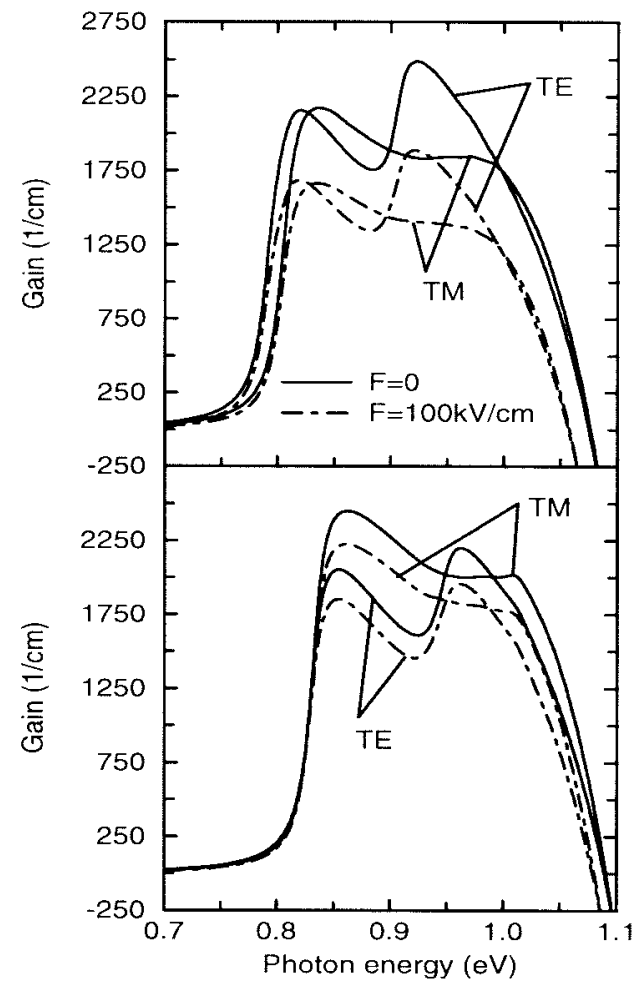

Fig. 10. TE and TM gain spectra for different applied fields of the $\mathrm{In}_{0.53} \mathrm{Ga}_{0.47} \mathrm{As}-\mathrm{InP}$ interdiffused QW with $L_{z}=120 \AA$ and a carrier density of $9 \times 10^{12} \mathrm{~cm}^{-2}$. (a) $t=0$ h. (b) $t=0.8 \mathrm{~h}$. $F=0$ (solid line) and $F=100 \mathrm{kV} / \mathrm{cm}$ (dashed line).

independence is an important issue for optical communications since fibers do not maintain the polarization state of an input optical beam. Therefore, polarization-independent gain is essential for polarization-independent optical amplifiers for use in optical communication.

\section{CONCLUSION}

In this paper, polarization-independent-interdiffused QW optical gain using interdiffusion has been investigated theoretically in $\mathrm{In}_{0.53} \mathrm{Ga}_{0.47}$ As-InP and $\mathrm{In}_{0.51} \mathrm{Ga}_{0.49} \mathrm{As}-\mathrm{InP}$ interdiffused QW structures. Taking into account free-carrier screening effects, the transition energy increases as compared to that without the carrier effects, and the blue shift is enhanced when the applied field is increased. The increasing transition energy is dominated by increasing the hole subband. With the consideration of carrier effects, the gain magnitude of an interdiffused QW slightly reduces for $F=0$ and increases when $F$ increases, in comparison to the corresponding case without carrier effects.

Polarization-independent gain can be obtained for these interdiffused QW structures over a wavelength range of $10 \mathrm{meV}$. The required time for polarization-independent gain is shorter than that of polarization-independent transition. When the carrier density increases, the polarization-independent gain increases. The maximum polarization-independent gain is 1800 $\mathrm{cm}^{-1}$ for a carrier density of $9 \times 10^{-12} \mathrm{~cm}^{-2}$. The optimized $\mathrm{In}_{0.51} \mathrm{Ga}_{0.49} \mathrm{As}-\mathrm{InP}$ interdiffused QW with $L_{z}=140 \AA$ and $t=0.1 \mathrm{~h}$ can operate at $0.8 \mathrm{eV}(1.55 \mu \mathrm{m})$ with polarizationindependent gain of $1000 \mathrm{~cm}^{-1}$, while the differential gain and the linewidth enhancement factor of the polarizationindependent interdiffused QW reduces and increases, respectively, without a significant degradation. For the as-grown lattice-matched QW structure, all the required tensile strain for the polarization independence can be generated using interdiffusion. This indicates the possibility that polarizationindependent longitudinal-type optical devices can be fabricated using a lattice-matched InGaAsP QW structure and interdiffusion technology.

The highest gain peak of the QW can be varied from the TE mode to the TM mode using interdiffusion. When $t$ increases, the TE gain peak which is the largest at $t=0$ reduces and the TM gain peak increases over the TE peak when $t \geq 0.5 \mathrm{~h}$ due to the increase of the interdiffusion-induced shear tensile strain in the well. Moreover, for InGaAsP QW lasers, the threshold current can be increased for TE mode operation and reduced for TM mode operation using interdiffusion.

\section{REFERENCES}

[1] M. Asada, A. Kameyama, and Y. Suematsu, "Gain and intervalence band absorption in quantum-well lasers," IEEE J. Quantum Electron., vol. QE-20, pp. 745-753, 1984.

[2] K. Margari, M. Okarmoto, H. Yasaka, K. Sato, Y Noguchi, and O. Mikami, "Polarization insensitive traveling wave type amplifier using strained multiple quantum well structure," IEEE Photon. Technol. Lett. vol. 2, pp. 556-558, 1990.

[3] J. J. He, S. Charbonnneau, P. J. Poole, G. C. Aers, Y. Feng, E. S Koteles, R. D. Goldberg, and I. V. Mitchell, "Polarization insensitive InGaAs/InGaAsP/InP amplifier using quantum well intermixing," Appl. Phys. Lett., vol. 69, pp. 562-564, 1996.

[4] T. Aizawa, K. G. Ravikumar, S. Suzaki, T. Watanabe, and R. Yamanchi, "Polarization-independent quantum-confined stark effect in an InGaAs/InP tensile-strained quantum well," IEEE J. Quantum Electron., vol. 30, pp. 585-592, 1994.

[5] W. C. H. Choy, E. H. Li, and J. Micallef, "The polarization insensitive electro-absorptive and -refractive modulation by utilizing InGaAsP/InP interdiffused quantum well," IEEE J. Quantum Electron., vol. 33, pp. 1316-1322, 1997.

[6] H. Venghaus and C. M. Weinert, "Polarization independent Mach-Zehnder interferometer on III-V semiconductor," IEEE Photon. Technol. Lett., vol. 5, pp. 1213-1216, 1993.

[7] T. Fujii, M. Sugawara, S. Yazaki, and K. Nakajima, "Macroscopic mechanism of group $\mathrm{V}$ interdiffusion in undoped InGaAs/InP quantum wells grown by MOVPE," J. Cryst. Growth, vol. 105, pp. 348-352, 1990.

[8] K. Mukai, M. Sugawara, and S. Yamazaki, "Interdiffusion process in lattice-matched $\operatorname{In}_{x} \mathrm{Ga}_{1-x} \mathrm{As}_{y} \mathrm{P}_{1-y} / \mathrm{InP}$ and $\mathrm{GaAs} / \mathrm{Al}_{x} \mathrm{Ga}_{1-x}$ As quantum well," Phys. Rev. B, vol. 50, pp. 2273-2282, 1994.

[9] J. M. Luttinger and W. Kohn, "Motion of electrons and holes in perturbed periodic fields," Phys. Rev., vol. 97, pp. 869-883, 1955.

[10] J. Micallef, E. H. Li, and B. L. Weiss, "Effect of interdiffusion on the sub-band-edge structure of $\operatorname{In}_{0.53} \mathrm{Ga}_{0.47} \mathrm{As} / \mathrm{InP}$ single quantum well," J. Appl. Phys., vol. 73, pp. 7524-7532, 1993.

[11] P. Lawaetz, "Valance-band parameters in cubic semiconductors," Phys. Rev. B, vol. 4, pp. 3460-3467, 1971.

[12] M. Altarelli, U. Ekenberg, and A. Fasolino, "Calculations of hole subbands in semiconductor quantum wells and superlattices," Phys. Rev. $B$, vol. 32, pp. 5138-5143, 1985.

[13] A. Twardowski and C. Hermann, "Variational calculation of polarization of quantum-well photoluminescence," Phys. Rev. B, vol. 35, pp 8144-8153, 1987.

[14] D. A. Broido and L. J. Sham, "Effective masses of holes at GaAsAlGaAs heterojunctions," Phys. Rev. B, vol. 31, pp. 888-892, 1985.

[15] D. Ahn and S. L. Chuang, "Valence band mixing effects on the gain and the refractive index change of quantum well lasers," J. Appl. Phys., vol. 64, pp. 4056-4064, 1988.

[16] W. C. H. Choy and E. H. Li, "Applications of interdiffused AlGaAs/GaAs quantum well in electro-absorptive modulation," IEEE J. Quantum Electron., vol. 34, pp. 1162-1170, 1998.

[17] G. Bastard and J. A. Brum, "Electronic states in semiconductors het erostructures," IEEE J. Quantum Electron., vol. QE-22, pp. 1625-1644, 
1986.

[18] D. J. BenDaniel and C. B. Duke, "Space-charge effects on electron tunneling," Phys. Rev., vol. 152, pp. 683-692, 1966.

[19] S. Seki, T. Yamanaka, W. Lui, Y. Yoshikuni, and K. Yokoyama, "Theoretical analysis of pure effects of strain and quantum confinement on differential gain in InGaAsP/InP strained-layer quantum-well lasers,' IEEE J. Quantum Electron., vol. 30, pp. 500-510, 1994.

[20] J. J. He, private communication.

Wallace C. H. Choy (S'98) received the B.Sc. (1st class) degree in applied physics from the Baptist University of Hong Kong with scholastic award in 1994 and the M.Phil. degree in electronic engineering from the University of Hong Kong in 1996. His thesis was concerned with the modeling of optical properties of interdiffusion-modified quantum-well structures for modulation devices. He is currently working toward the Ph.D. degree in electronic engineering at the University of Surrey, U.K.

He currently holds a Croucher Foundation Scholarship. His main research topics include the influence of applied electric fields, injected carriers and acoustic waves in quantum-well materials, and electrooptic and acoustooptic devices for optoelectronic applications.

Mr. Choy was awarded the Sir Edward Youde Memorial Fellowship and a Postgraduate Studentship award while performing his graduate work.

E. Herbert Li (S'87-M'88-SM'95) received the M.Phil. degree in applied mathematics and electronic engineering from the University of Hong Kong and the Ph.D. degree in electronic engineering from the University of Surrey, U.K.

He worked for the Kirsten Aeronautical Laboratory, Seattle, WA (1979-1980). He then joined KK Engineering Company, Hong Kong, as an Engineer (1981-1986) and Micro Systems, Hong Kong, as a Manager (1986-1988). He was a Member of Faculty at the City University of Hong Kong (1988-1990), and he joined the National Ion Implantation Facility at the Department of Electrical Engineering, University of Surrey, from 1990 to 1993. He is currently a Member of Faculty and Leader of the Optoelectronics Group, which consists of a team of ten researchers, at the Department of Electrical and Electronic Engineering, University of Hong Kong (1994-present). He is also an Adjunct Professor of the Institute of Semiconductors, Shandong Normal University, China, and a Visiting Faculty Member at the University of Waterloo, Canada. His current research interests are mainly concerned with optoelectronic device fabrication, modeling, and characterization, and, in particular, interdiffusion-induced modification of quantum-well structures for the advanced performance and integration of optoelectronic devices. Since 1990, he has published over 100 international technical papers, two book chapters, and three books in the above areas. He served on both the Technical Program and International Advisory Committees of OECC in 1996 (Japan) and 1997 (Korea). He is an Editor of the International Journal of Optoelectronics. His biography is published in Who's Who in Science and Engineering.

Dr. Li was a "Distinguished Lecturer" of the IEEE Electron Device Society (ED-S) for 1997, and he is an AdCom ex officio member and EDS Meeting Committee member of the IEEE. He received the J. Langham Thompson Premium Prize in 1992 from the Institution of Electrical Engineers (U.K.) and the Distinguished Pioneering Projects Award in 1989 from HKCSS (Hong Kong).
Michael C. Y. Chan, photograph and biography not available at the time of publication.

Bernard L. Weiss (S'72-M'74-SM'94) received the B.Sc. degree in electrical engineering and the Ph.D. degree in solid-state electronics from the University of Newcastle upon Tyne, U.K., in 1971 and 1974, respectively.

He was awarded an SRC Personal Post-Doctoral Research Fellowship for the study of anodic oxides on GaAs in 1975, and in 1977 he moved to University College London as a Research Fellow, where he worked on acoustic nondestructive testing. In 1979, he was appointed a Lecturer in Electronic Engineering at the University of Surrey, U.K., where he set up research in integrated optical devices in $\mathrm{LiNbO}_{3}$. He was promoted to Senior Lecturer, Reader, and Professor in 1986, 1993, and 1996, respectively. His research is concerned with modeling the optical properties of III-V semiconductor quantum-well structures and devices and optoelectronic devices in silicon-based materials and photosensitivity in glass. In 1991, he spent six months sabbatical leave at the University of Cincinnati, Cincinnati, $\mathrm{OH}$, in 1994-1995, he was a DFG Visiting Professor at the Technische Hoschschule Darmstadt, Germany, and he is an Honorary Professor at the University of Hong Kong.

Dr. Weiss is a fellow of the Institution of Electrical Engineers (IEE) U.K. and the Institute of Physics (U.K.). He is Chair of the IEE Scholarships Committee, a member of several other IEE committees, and Editor of the IEE EMIS book series. He was awarded the Medal of the University of Warsaw for his contributions to the TEMPUS project. 